\section{Contribuição da Glicemia Pós-Desjejum Para o Controle Glicêmico do Paciente Com Diabetes Melito Tipo 2}

\section{RESUMO}

Estudos epidemiológicos observaram que glicemias pós-prandiais (GPPs) elevadas são fator principal na ocorrência de doenças cardiovasculares. Sabe-se que a hemoglobina glicada $\left(\mathrm{HbA}_{1 \mathrm{c}}\right)$ reflete a glicemia média dos últimos 2-3 meses, entretanto é controversa a contribuição relativa da glicemia de jejum (GJ) e GPP para o valor da $\mathrm{HbA}_{1 \mathrm{c}}$. Objetivo: Avaliar a contribuição da GJ e GPPs para o valor da $\mathrm{HbA} \mathrm{Ac}_{1 \mathrm{C}}$ em pacientes com diabetes melito tipo 2 (DM2). Métodos: Participaram 53 indivíduos com DM2, estáveis e em tratamento com antidiabéticos orais $(n=27)$ e/ou insulina $(n=26)$. Cada paciente comparecia a 3 visitas a intervalos de 2 meses. Em cada visita era medida a GJ, as GPPs (2h pós-desjejum: GPD e pósalmoço: GPA) e a $\mathrm{HbA}_{1 \mathrm{c}}$, sendo fornecido o desjejum e o almoço segundo seus hábitos alimentares. Mediu-se a glicose plasmática pela glicoseoxidase e a $\mathrm{HbA}_{1 \mathrm{c}}$, pela cromatografia de troca iônica. Realizou-se a análise das associações pelo coeficiente de correlação de Spearman, com $\mathrm{P}<0,05$. Resultados: $\mathrm{A} \mathrm{HbA} 1 \mathrm{c}$ correlacionou-se melhor em cada visita ao longo do estudo com a GPD $(r$ : 0,66-0,48), a glicemia média (r: $0,64-0,41)$, a área abaixo da curva glicêmica $(r: 0,64-0,46)$ e a GPP média (r: 0,59-0,41). Conclusões: A GPD mostrou-se um parâmetro eficaz adicional no monitoramento glicêmico dos pacientes com DM2. (Arq Bras Endocrinol Metab 2006;50/1:53-59)

Descritores: Glicemia pós-prandial; Glicemia pós-desjejum; Hemoglobina glicada; Diabetes melito tipo 2

\section{ABSTRACT}

Contribution of Post-breakfast Plasma Glucose to the Glycemic Control of Type 2 Diabetic Patients.

Epidemiological studies have documented that postprandial hyperglycemia is the main risk factor for cardiovascular diseases. It has been established that glycated hemoglobin $\left(\mathrm{HbA}_{1 \mathrm{C}}\right)$ provides an integrated measure of plasma glucose (PG) of the last 2-3 months. However, the relative contribution of fasting PG (FPG) and postprandial PG (PPG) to the $\mathrm{HbA}_{1 \mathrm{C}}$ value is controversial. Objective: To evaluate FPG and PPG contributions to the $\mathrm{HbA}_{1 \mathrm{C}}$ value in patients with type 2 diabetes mellitus (DM2). Methods: 53 subjects with stable DM2 were studied. They were treated with oral anti-diabetic agents $(n=27)$ and/or insulin $(n=26)$. Each subject went to 3 visits at 2-month-intervals. On each visit, FPG, PPG (2h after breakfast and lunch), and $\mathrm{HbA}_{1 \mathrm{C}}$ were measured and we provided breakfast and lunch according to their meal habits. PG was measured by glucose-oxidase and $\mathrm{HbA}_{1 \mathrm{C}}$ by ion-exchange chromatography. Statistical analysis was performed by correlation coefficients at a $<0.05 P$ value. Results: Correlations were stronger between $\mathrm{HbA}_{1 \mathrm{C}}$ and post-breakfast $\mathrm{PG}$ ( $\mathrm{r}$ : $0.66-0.48)$, mean FPG ( $r: 0.64-0.41)$, glucose area under the curve ( $r$ : 0.64-0.46), and mean PPG (r: 0.59-0.41). Conclusions: Measurement of post-breakfast PG showed to be another valuable tool for type 2 diabetic glucose control monitoring. (Arq Bras Endocrinol Metab 2006;50/1:53-59)

Keywords: Postprandial plasma glucose; Post-breakfast plasma glucose; Glycated hemoglobin; Type 2 diabetes mellitus artigo original

\author{
Maria Salete Sartori \\ Flávio Fervari Aragon \\ Carlos Roberto Padovani \\ Walkyria de P. Pimenta
}

Departamento de Clinica Médica, Faculdade de Medicina de Botucatu (MSS, WPP), e

Departamento de Bioestatistica, Instituto de Biociências de Botucatu (FFA, CRP), UNESP, Botucatu, SP.
Recebido em 10/05/05

Revisado em 18/08/05 Aceito em 04/11/05 
$\mathbf{O}^{2}$ Diabetes melito TIPO 2 (DM2) é uma das doenças metabólicas mais comuns. Estudo epidemiológico brasileiro verificou prevalência de 7,6\% de diabetes na população urbana entre 30 e 69 anos de idade (1). Tanto em países desenvolvidos como em desenvolvimento, o número de indivíduos com DM2 está aumentando rapidamente, prevendo-se sua duplicação nos próximos 20 anos (2). As complicações degenerativas neuro-vasculares do DM2, principalmente as doenças cardiovasculares (DCVs), diminuem a qualidade de vida do paciente diabético e constituem um ônus para a sociedade.

A hiperglicemia determina a glicação de proteínas com liberação de vários fatores de inflamação (citocinas) e de estresse oxidativo (radicais oxidantes) e diminuição do metabolismo de substratos com redução de ATP, causando degeneração e disfunção vasculares (3-5). A hiperglicemia associada a outros fatores freqüentemente presentes no DM2, tais como dislipidemia e hipertensão arterial, tornam o paciente com DM2 mais vulnerável à DCV (2).

A meta-análise realizada por Coutinho e cols. (6) compreendeu 20 estudos envolvendo 95.783 indivíduos não diabéticos e 3.707 eventos cardiovasculares durante um período de seguimento de 12,4 anos. Verificaram que ambas, a glicemia de jejum (GJ) e a glicemia de 2 horas após uma carga oral de glicose (G2h), associaram-se independentemente com risco aumentado para DCVs. Todavia, vários outros estudos in vitro (7) e no homem, prospectivos e observacionais (8-10) ou interventivos (11-13), demonstraram o papel maior da glicemia pós-prandial (GPP) ou da G2h elevada no risco para ocorrência de morte e DCVs. Destes estudos observa-se que, precocemente na evolução para o DM2, como na fase de tolerância à glicose diminuída, já ocorre intensificação do processo de aterosclerose $(8,14,15)$. Tal fato dar-se-ia pela elevação da GPP resultar principalmente da diminuição da $1^{a}$ fase de secreção de insulina, que é um dos primeiros defeitos a aparecer naqueles que se tornam diabéticos (16). Ainda, depreende-se que a hiperglicemia pós-prandial ocorrendo juntamente com outros distúrbios metabólicos, como a elevação dos triglicérides, deve favorecer o processo de aterosclerose (16-18). O estudo que forneceu a evidência mais convincente de risco aumentado para DCV relacionado à G2h diagnóstica de diabetes é o DECODE (10). Trata-se de estudo europeu multicêntrico envolvendo mais de 25.000 indivíduos. Observa-se que dentro de cada categoria de GJ (<110, 110-125, $\geq 126 \mathrm{mg} / \mathrm{dL})$ o risco de morte (por DCVs) aumentava linearmente através das categorias de G2h (<140, 140-199, $\geq 200$ $\mathrm{mg} / \mathrm{dL}$ ), enquanto o inverso não ocorreu. Os estudos epidemiológicos que visaram ao controle da hiperglicemia pós-prandial, seja com insulina de ação rápida $(11,12)$ ou com antidiabéticos orais como a acarbose (13), mostraram benefício na redução da ocorrência dos eventos cardiovasculares.

Assim, embora não esteja estabelecido que o controle da hiperglicemia pós-prandial determinará diminuição da freqüência de DCV nos diabéticos, cada vez mais compreende-se a importância do controle das GJs e GPPs no DM2. Atualmente, apenas no diabetes melito gestacional ficou demonstrado, por De Venciana e cols. (19), que o controle glicêmico pós-prandial vs. o pré-prandial determina maior queda dos valores de hemoglobina glicada $\left(\mathrm{HbA}_{\mathrm{lC}}\right)$ e melhores resultados fetais.

Dever-se-ia então, na avaliação do controle glicêmico do paciente com DM2, medir, além da GJ e da $\mathrm{HbA}_{\mathrm{lc}}$, as GPPs. Buscando maior facilidade e eficácia e menor ônus financeiro no acompanhamento destes pacientes, têm-se investigado a contribuição relativa da GJ e da GPP para o valor da $\mathrm{HbA}_{1 \mathrm{C}}$ e a importância de cada uma das três principais medidas de GPP. Os resultados obtidos são controversos.

Avignon e cols. (20) foram os primeiros a chamar a atenção para o fato de as glicemias não de jejum, isto é, de duas e cinco horas após o almoço, se associarem mais ao valor da $\mathrm{HbA}_{1 \mathrm{C}}$ em pacientes com DM2 em tratamento com dieta e antidiabético oral. Pouco depois, no mesmo tipo de paciente, todavia pertencente a outro grupo populacional, Soonthorpun e cols. (21) observaram que a glicemia de duas horas após uma refeição de carboidratos era a que melhor se associava à $\mathrm{HbA}_{\mathrm{lC}}$. Por outro lado, Bonora e cols. (22), em estudo amplo, com diabéticos tipo $2 \mathrm{da}$ Clínica de Diabetes de Verona, em tratamento com dieta associada geralmente a um antidiabético oral, observaram por meio das dosagens de perfil glicêmico e de $\mathrm{HbA}_{\mathrm{lC}}$, colhidos em casa ou no hospital ambulatorialmente ou na enfermaria, que a $\mathrm{HbA}_{\mathrm{lC}}$ forneceu pouca informação sobre os níveis de GPP e nenhuma sobre a variação glicêmica ocasionada pelas refeições. O estudo de Monnier e cols. (23) é conciliador. Observaram que em diabéticos tipo 2 estáveis, as GPPs tinham contribuição maior para o valor da $\mathrm{HbA}_{\mathrm{lC}}$ naqueles com hiperglicemia leve ou moderada, enquanto as GJs o tinham naqueles com mau controle glicêmico.

Assim, nosso objetivo foi avaliar a contribuição da GJ e de GPPs no valor da $\mathrm{HbA}_{\mathrm{lC}}$ em pacientes com DM2, acompanhados ambulatorialmente num Hospital Universitário, estáveis e moderadamente controla- 
dos por meio de antidiabéticos orais e/ou insulina. Os participantes deviam estar em bom estado geral e não apresentar doença associada ou complicação crônica avançadas.

\section{MÉTODOS}

Participaram do estudo 53 indivíduos com DM2, cujas principais características clínicas são apresentadas na tabela 1

Após a aprovação do projeto pelo Comitê de Ética em Pesquisa de nossa Instituição, cada participante foi esclarecido e deu seu consentimento livre e por escrito. A seguir, cada um era entrevistado para obtenção de seu recordatório alimentar e agendadas três visitas a intervalo de dois meses entre cada uma. Solicitou-se aos pacientes que mantivessem suas atividades, hábitos alimentares e tratamento durante o estudo.

Para cada uma das visitas o paciente comparecia ao Laboratório às $7 \mathrm{~h} 00$, tendo observado jejum noturno (8-12 h) e trazendo urina das $24 \mathrm{~h}$ anteriores para posterior dosagem da glicose (GU). Coletava-se uma amostra de sangue venoso para dosagem de glicose (GJ) e da $\mathrm{HbA}_{1 \mathrm{c}}$. A seguir, os pacientes recebiam os medicamentos habituais e o desjejum (7h30). Depois, permaneciam em ambiente hospitalar desenvolvendo alguma atividade de entretenimento e sem ingestão de qualquer alimento. Duas horas após, retornavam ao Laboratório para coleta de sangue para dosagem de glicose (GPD) e depois voltavam a observar a conduta prévia. Às 11 h30 recebiam o almoço e após, aguardavam duas horas para a coleta da glicemia pós-almoço (GPA). Assim, estava terminada a visita e o participante era conduzido ao ambulatório para o atendimento médico. As duas refeições oferecidas eram preparadas pelo Serviço de Nutrição e Dietética de nosso Hospital com base no recordatório alimentar. Tratava-se de ingestão calórica média diária de $1.740 \mathrm{kcal}$, das quais $20 \%$ correspondiam ao desjejum e $40 \%$ ao almoço e de

Tabela 1. Características clínicas.

\begin{tabular}{lc}
\hline $\mathrm{N}$ & 53 \\
Sexo (M/F) & $16 / 37$ \\
Idade (anos)* & $54 \pm 10$ \\
IMC+ (kg/m²)* $^{*}$ & $29,5 \pm 2,9$ \\
Duração diabetes (anos)* & $7 \pm 5$ \\
Tratamento: Sem insulina & 27 \\
\multicolumn{1}{c}{ Com Insulina } & 26 \\
Hipertensão arterial** & 71 \\
Dislipidemia** & 51 \\
Complicações crônicas** $^{*}$ & 49 \\
\hline
\end{tabular}

+ Índice de Massa Corporal; * Mediana \pm semi-amplitude interquartilica; ${ }^{* *}$ Porcentagem
$21 \mathrm{~g}$ de fibras. Cada refeição era composta de $50 \%$ carboidratos, 19\% proteínas e 31\% lipídios.

A glicose plasmática e a urinária foram dosadas pelo método da glicose oxidase. A medida da $\mathrm{HbA}_{1 \mathrm{C}}$ foi realizada pelo método da cromatografia de troca iônica utilizando-se os kits comerciais da Labtest, MG, Brasil (sendo $80 \%$ do valor da $\mathrm{HbA}_{1}$ obtido). Os valores da $\mathrm{HbA}_{1 \mathrm{C}}$ de 10 indivíduos adultos e saudáveis de nosso meio variaram de 6,4 a $7,8 \%$, com média \pm DP de $7,1 \pm 0,4 \%$. Valores estes semelhantes à faixa de normalidade relatada para o método $(5,3-8,0 \%)$. O erro intraensaio foi de $1,87 \%$.

As medidas descritivas dos parâmetros de controle glicêmico foram analisadas pelo teste nãoparamétrico para medidas repetidas de Friedman (24). $\mathrm{Na}$ avaliação da estabilidade glicêmica, empregaram-se os testes não-paramétricos para medidas repetidas de Wilcoxon e de aderência do qui-quadrado para uma amostra com proporções esperadas iguais (24). Nas análises de associação utilizou-se o coeficiente de correlação de Spearman (24). No estudo das glicemias dos três momentos de cada visita como parâmetro preditivo dos valores da $\mathrm{HbA}_{\mathrm{lC}}$, empregaram-se as medidas de sensibilidade, especificidade e valores preditivos (24). Considerou-se como significantes os valores de $\mathrm{P}<0,05$.

\section{RESULTADOS}

Nas três avaliações realizadas num período de quatro meses, os participantes com DM2 apresentaram controle glicêmico satisfatório e estável (tabela 2), sendo a variação do valor da $\mathrm{HbA}_{1 \mathrm{C}}$ durante cada intervalo de tempo de dois meses menor que $0,5 \%$.

As correlações mais fortes ocorreram entre a $\mathrm{HbA}_{\mathrm{lC}}$ e a GPD, a glicemia média de cada visita e a resposta glicêmica total de cada visita como área abaixo da curva glicêmica (tabela 3 ). Também foi forte a correlação entre a GPP média de cada visita e a $\mathrm{HbA}_{\mathrm{lC}}$ (tabela 3). Menos intensa mas presente durante todo período observacional foi a correlação entre a $\mathrm{HbA}_{1 \mathrm{C}}$ e a variação glicêmica após o desjejum. Por outro lado, menos intensas e presentes apenas nas duas primeiras avaliações foram as correlações entre a $\mathrm{HbA}_{1 \mathrm{C}}$ e as GJ e GPA, e não houve correlação entre a $\mathrm{HbA}_{\mathrm{lC}}$ e a variação da glicemia média de cada visita e a variação glicêmica após o almoço (tabela 3 ).

Considerando-se como índices de controle glicêmico satisfatório os valores glicêmicos menores que $110 \mathrm{mg} / \mathrm{dL}$ para o período de jejum e menores que $140 \mathrm{mg} / \mathrm{dL}$ para os períodos pós-prandiais, foi 
Tabela 2. Controle glicêmico ao longo do estudo.

\begin{tabular}{lcccc}
\hline & \multicolumn{4}{c}{ Visita } \\
\cline { 2 - 5 } & Primeira & Segunda & Terceira & P \\
\hline Glicemia jejum $(\mathrm{mg} / \mathrm{dL})^{*}$ & $118 \pm 26$ & $114 \pm 19$ & $115 \pm 25$ & $\mathrm{NS}$ \\
Glicemia pós-desjejum (mg/dL) & $145 \pm 43$ & $139 \pm 55$ & $139 \pm 44$ & $\mathrm{NS}$ \\
Glicemia pós-almoço (mg/dL) & $128 \pm 40$ & $137 \pm 47$ & $127 \pm 36$ & $\mathrm{NS}$ \\
Glicosúria (g/24h) & $0 \pm 0,18$ & $0 \pm 0,18$ & $0 \pm 0,03$ & $\mathrm{NS}$ \\
Hemoglobina glicada (HbAlC-\%) & $6,5 \pm 1,4$ & $6,5 \pm 1,1$ & $6,6 \pm 1,0$ & $\mathrm{NS}$ \\
\hline
\end{tabular}

* Mediana \pm semi-amplitude interquartílica

Tabela 3. Correlações entre a hemoglobina glicada ( $\mathrm{HbAlC}-\%)$ e as glicemias (mg/dL): jejum (GJ), pós-desjejum (GPD), pós-almoço (GPA), média (GM) e pós-prandial média (GPPM); a área abaixo da curva glicêmica (AACG-mg.h/dL); a variação da GM acima da GJ (DGM-mg/dL) e das GPD e GPA acima da GJ (DGPD-mg/dL e DGPA-mg/dL, respectivamente) dos participantes ao longo do estudo.

\begin{tabular}{lcccccc}
\hline & \multicolumn{7}{c}{ HbA $_{1 C}$} \\
\cline { 2 - 7 } & \multicolumn{7}{c}{ Visita } \\
\cline { 2 - 7 } & Primeira & $\mathbf{P}$ & Segunda & $\mathbf{P}$ & Terceira & $\mathbf{P}$ \\
\hline GJ & 0,55 & $<0,0001$ & 0,38 & $<0,005$ & 0,29 & NS \\
GPD & 0,66 & $<0,0001$ & 0,49 & $<0,0005$ & 0,48 & $<0,001$ \\
GPA & 0,42 & $<0,0001$ & 0,33 & $<0,01$ & 0,25 & NS \\
GM & 0,64 & $<0,0001$ & 0,50 & $<0,0005$ & 0,41 & $<0,005$ \\
GPPM & 0,59 & $<0,0001$ & 0,47 & $<0,0005$ & 0,41 & $<0,005$ \\
AACG & 0,64 & $<0,0001$ & 0,50 & $<0,001$ & 0,46 & $<0,005$ \\
$\Delta$ GM & 0,26 & NS & 0,21 & NS & 0,25 & NS \\
$\Delta$ GPD & 0,44 & $<0,001$ & 0,33 & $<0,01$ & 0,31 & $<0,05$ \\
$\Delta$ GPA & 0,10 & NS & 0,11 & NS & 0,15 & NS \\
\hline
\end{tabular}

calculada a sensibilidade, a especificidade e os valores preditivos para os controles glicêmicos satisfatório $\left(\mathrm{HbA}_{1 \mathrm{C}}<7,0 \%\right)$ e insatisfatório $\left(\mathrm{HbA}_{\mathrm{lC}} \geq 7,0 \%\right)$ para as três medidas glicêmicas e para a glicemia média, ao longo do estudo (tabela 4). A GPD mostrou sensibilidade entre 73 e $85 \%$, especificidade entre 68 e $76 \%$ e valores preditivos positivo e negativo entre 62 e $78 \%$ e 78 e $88 \%$, respectivamente. Assim, entre os três parâmetros glicêmicos foi a que teve melhor desempenho, o qual foi comparável ao da glicemia média.

\section{DISCUSSÃO}

Os 53 pacientes com DM2 participantes deste estudo possuíam características clínicas freqüentemente observadas nesta classe de diabetes após tempo mediano de doença de aproximadamente sete anos: idade mediana acima dos 50 anos, sobrepeso, associação com hipertensão arterial e dislipidemia, presença de uma ou mais complicações crônicas do diabetes e necessidade de utilização de insulina para o controle glicêmico em metade do grupo.
Os valores mais elevados da GPD em relação aos da GPA também foram observados por Monnier e cols. (25) em estudo semelhante. Tal resultado deverse-ia à inversão da variação circadiana da glicemia, isto é, glicemias mais elevadas no período matutino que vespertino, relatada em pacientes diabéticos desde os estudos iniciais, e cuja etiopatogênese ainda é controversa $(26,27)$.

Durante o período de quatro meses de avaliações do controle glicêmico, os pacientes mantiveramse estáveis, o que permitiu as análises de correlação entre os parâmetros glicêmicos e os valores da $\mathrm{HbA}_{1 \mathrm{C}}$. Das três glicemias medidas, a GPD foi a que mostrou correlação mais forte e constante com a $\mathrm{HbA}_{1 \mathrm{C}}$. Um dos estudos pioneiros que chamou a atenção para a importância da contribuição da concentração das GPPs no valor da $\mathrm{HbA}_{1 \mathrm{C}}$ foi o de Avignon e cols. (20). Estes autores observaram melhor associação entre as glicemias não-jejum ( 2 e 5 horas após o almoço) que a de jejum, e a $\mathrm{HbA}_{1 \mathrm{C}}$. Tal resultado seria explicado pelo período pós-prandial compreender mais amplamente os distúrbios metabólicos próprios dos pacientes com DM2 que o de jejum. Em 35 diabéticos tipo 2 
Tabela 4. Medidas de sensibilidade (S), especificidade (E) e valores preditivos (VP) para os controles glicêmicos satisfatório $\left(\mathrm{HbA}_{1 \mathrm{C}}<7,0 \%\right)$ e insatisfatório $\left(\mathrm{HbA}_{1 \mathrm{C}} \geq 7,0 \%\right)$ para as três medidas glicêmicas e para a glicemia média dos participantes, em cada visita ao longo do estudo.

\begin{tabular}{llllll}
\hline Glicemia (mg/dL) & Visita & S (\%) & E (\%) & VP+ (\%) & VP- (\%) \\
\hline Jejum & Primeira & 80,95 & 46,88 & 50,00 & 78,95 \\
Pós-Desjejum & & 85,11 & 68,75 & 64,29 & 88,00 \\
Pós-Almoço & & 66,66 & 75,00 & 63,64 & 77,42 \\
Média & & 80,95 & 81,25 & 73,91 & 86,66 \\
\hline Jejum & & 66,67 & 44,83 & 50,00 & 61,91 \\
Pós-Desjejum & Segunda & 79,17 & 75,86 & 73,08 & 81,48 \\
Pós-Almoço & & 66,67 & 70,37 & 61,54 & 70,37 \\
Média & & 75,00 & 79,31 & 75,00 & 79,31 \\
\hline Jejum & & 63,64 & 51,61 & 48,28 & 66,67 \\
Pós-Desjejum & Terceira & 72,73 & 67,74 & 61,54 & 77,78 \\
Pós-Almoço & & 45,45 & 67,74 & 50,00 & 63,64 \\
Média & & 54,54 & 70,97 & 54,43 & 68,75 \\
\hline
\end{tabular}

estáveis, Soonthorpun e cols. (21) observaram que a glicemia de $2 \mathrm{~h}$ após uma refeição-teste à base de carboidratos foi a que mais se correlacionou com a $\mathrm{HbA}_{\mathrm{lC}}$, explicando em $1 / 3$ destes pacientes os valores de $\mathrm{HbA}_{1 \mathrm{C}} \geq 7,0 \%$. Em estudo semelhante ao nosso, exceto pela avaliação das glicemias ter sido realizada pelo automonitoramento em casa (glicemias capilares) no período das $8 \mathrm{~h} 00$ às $16 \mathrm{~h} 00$, Lerman-Garber e cols. (28) observaram que a melhor correlação com a $\mathrm{HbA}_{\mathrm{lC}}$ se dava com a glicemia pós-café. Trovati e cols. (29) e Bouma e cols. (30), em número grande de diabéticos tipo 2 , verificaram que a $\mathrm{GJ}$ não refletia adequadamente o controle glicêmico pelos valores da $\mathrm{HbA}_{\mathrm{lC}}$, chamando a atenção para a necessidade de se avaliar também as GPPs nestes pacientes. No presente estudo, o resultado da GPD ter-se correlacionado melhor que a GPA com a $\mathrm{HbA}_{\mathrm{lC}}$ poderia ser devido a seus valores mais elevados.

Por outro lado, há estudos que apóiam o posicionamento da American Diabetes Association (31) de que a GJ é melhor que as GPPs em predizer o valor da $\mathrm{HbA}_{1 \mathrm{C}}$, especialmente nos pacientes com DM2. Entre estes estudos há os de Schmitz e cols. (32) e de Bonora e cols. (22), que envolveram diabéticos tipo $2 \mathrm{com}$ maior descontrole glicêmico que os participantes de nosso estudo, e os de Hillman e cols. $(33,34)$ com diabéticos tipo 1 sob tratamento insulínico intensivo. Tal explicação encontra apoio no estudo de Monnier e cols. (23). Estes autores observaram que a contribuição maior das GPPs ou das GJs para a hiperglicemia diária de pacientes com DM2 estava na dependência do grau do controle glicêmico ser satisfatório ou não, respectivamente.

Uma vez que o valor da $\mathrm{HbA}_{\mathrm{lC}}$ resulta da média das glicemias de um período anterior de 2-3 meses, a glicemia média de cada visita, mesmo sendo resultante de apenas três medidas, correlacionou-se bem com a $\mathrm{HbA}_{1 \mathrm{C}}$ durante o estudo. Vários estudos em diabéticos tipo 2 , seja por meio do perfil glicêmico de 4 a 6 medidas $(22,35)$, seja pelo automonitoramento glicêmico (28), ou mesmo em diabéticos tipo 1 , por glicemias capilares $(33,34)$, também observaram correlação entre a glicemia média diária e a $\mathrm{HbA}_{\mathrm{lC}}$. A importância da participação das GJs e GPPs fica expressa quando deixou de haver associação entre a $\mathrm{HbA}_{1 \mathrm{C}}$ e a variação glicêmica média acima do valor de jejum. O mesmo foi observado por Bonora e cols. (22) em estudo mais amplo.

Também evidente foi a correlação observada entre a $\mathrm{HbA}_{\mathrm{lC}}$ e a média das GPPs, como igualmente verificada por Schmitz e cols. (32), considerando inclusive o jantar em grupo de 19 diabéticos tipo 2 tratados apenas com dieta. Nesta associação pudemos demonstrar, mais uma vez, o papel predominante da elevação glicêmica após o desjejum, acima dos níveis glicêmicos basais, em relação àquela após o almoço. Por outro lado, Schmitz e cols. (32), apesar do resultado acima citado, e Bonora e cols. (22) não observaram contribuição independente e/ou significativa, respectivamente, da elevação glicêmica pós-refeições para o valor da $\mathrm{HbA}_{\mathrm{lC}}$. Comparativamente ao nosso estudo, o primeiro trabalho envolveu menor número de pacientes com DM2 apresentando pior grau de controle glicêmico, e a avaliação realizada compreendeu o automonitoramento glicêmico incluindo o período pós-jantar. O segundo estudo, embora tenha envolvido número muito grande de diabéticos tipo 2, cujo tratamento não compreendia a administração de insulina, $50 \%$ deles foram avaliados internados, com coleta de perfil glicêmico que se estendia até o período pós-jantar, encontrando-se estes pacientes em pior controle glicêmico que os que participaram de nosso estudo. 
A resposta glicêmica total em cada visita também se correlacionou significativamente com o respectivo valor da $\mathrm{HbA}_{1 \mathrm{C}}$, demonstrando o efeito integrador de todos os momentos glicêmicos para o resultado da $\mathrm{HbA}_{1 \mathrm{C}}$. O mesmo resultado foi obtido por Soonthorpun e cols. (21) em 35 diabéticos tipo 2 após a ingestão de uma refeição-teste à base de arroz.

Neste ponto devemos observar que em todas associações estudadas e que acabamos de comentar, nota-se um enfraquecimento das mesmas com o correr do tempo de observação. Talvez, isto se deva ao efeito da variabilidade biológica individual ao longo dos quatro meses nos valores da $\mathrm{HbA}_{1 \mathrm{C}}$ (36).

Entre as glicemias medidas em cada visita, a GPD foi a que apresentou valores mais elevados de sensibilidade, especificidade e valores preditivos positivo e negativo em relação à $\mathrm{HbA}_{1 \mathrm{C}}$, nas três visitas. Resultado semelhante foi relatado para a glicemia de 2 a 5 horas após o almoço (20), ou para a GJ $(30,37)$, ou ainda para os três momentos glicêmicos: jejum, pós-café e pós-almoço (28). Os diferentes momentos glicêmicos como melhores preditores do valor da $\mathrm{HbA}_{1 \mathrm{C}}$ relatados devem-se provavelmente às diferenças no delineamento dos estudos e principalmente aos diferentes valores de corte glicêmicos e da $\mathrm{HbA}_{1 \mathrm{C}}$ como definição de bom ou mau controle glicêmico.

A glicemia média de cada visita como parâmetro preditor do respectivo valor da $\mathrm{HbA}_{1 \mathrm{C}}$ foi semelhante à GPD. Da mesma forma que ocorre para os valores glicêmicos isolados, para a glicemia média os resultados são mais ou menos semelhantes entre os estudos. Em diabéticos tipo 1 a glicemia média capilar diária resultante do automonitoramento apresentou valores semelhantes aos nossos quanto à sensibilidade, especificidade e valor preditivo negativo $\left(\mathrm{HbA}_{\mathrm{lC}}<7,0 \%\right)$, segundo Hillman e cols. (33). Já Lerman-Garber e cols. (28) relataram maior sensibilidade/menor especificidade e menor valor preditivo positivo $\left(\mathrm{HbA}_{1 \mathrm{C}}>\right.$ $8,0 \%)$ que os nossos valores, para a glicemia média capilar diária resultante do automonitoramento por pacientes com DM2.

Concluindo, num grupo de pacientes com DM2 estáveis e satisfatoriamente controlados, observamos num período de quatro meses que: l) as GJ e GPPs contribuíram para o valor glicêmico médio e para a resposta glicêmica total, e ambos correlacionaram-se ao respectivo valor da $\mathrm{HbA}_{1 \mathrm{C}} ; 2$ ) isoladamente, a GPD (mesmo sua variação acima da GJ) mostrou correlação mais forte com a $\mathrm{HbA}_{1 \mathrm{C}}$ e ser o melhor parâmetro preditor do valor da $\mathrm{HbA}_{1 \mathrm{C}}$, sendo neste aspecto comparável à glicemia média. Assim, a medida da GPD pode fornecer um parâmetro adicional de interesse na avaliação do controle glicêmico de pacientes com DM2.

\section{REFERÊNCIAS}

1. Malerbi DA, Franco LJ, The Brazilian Cooperative Group on the Study of Diabetes Prevalence. Multicenter study of the prevalence of diabetes mellitus and impaired glucose tolerance in the urban Brazilian population aged 30-69 yr. Diabetes Care 1992; 15:1509-16.

2. Bonora $\mathrm{E}$, Muggeo M. Postprandial blood glucose as a risk factor for cardiovascular disease in Type II diabetes: The epidemiological evidence. Diabetologia 2001; 44:2107-14.

3. Brownlee M, Aiello LP, Friedman E, Vinik Al, Nesto RW, Boulton AJM. Complications of diabetes mellitus. In: Larsen PR, Kronenberg HM, Melmed S, Polonsky KS, editors. Williams textbook of endocrinology. $10^{\text {th }}$ ed. Philadelphia: Saunders; 2003. pp.1509-83.

4. Yan SF, Ramasamy R, Naka Y, Schmidt AM. Glycation, inflammation, and RAGE: A scaffold for the macrovascular complications of diabetes and beyond. Circ Res 2003:93:1159-69.

5. Ceriello A. Postprandial hyperglycemia and diabetes complications: Is it time to treat? Diabetes 2005:54: 1-7.

6. Coutinho M, Gerstein HC, Wang Y, Yusuf S. The relationship between glucose and incident cardiovascular events. A metaregression analysis of published data from 20 studies of 95,783 individuals followed for 12.4 years. Diabetes Care 1999;22:233-40.

7. Akbari CM, Saouaf R, Barnhill DF, Newman PA, LoGerfo FW, Veves A. Endothelium-dependent vasodilatation is impaired in both microcirculation and macrocirculation during acute hyperglycemia. J Vasc Surg 1998;28:68794.

8. Tominaga $\mathrm{M}$, Eguchi $\mathrm{H}$, Manaka $\mathrm{H}$, Igarashi $\mathrm{K}$, Kato $\mathrm{T}$, Seikikawa A. Impaired glucose tolerance is a risk factor for cardiovascular disease, but not impaired fasting glucose. The Funagata Diabetes Study. Diabetes Care 1999:22:920-4.

9. Rodrigues $\mathrm{BL}$, Lau N, Burchfield $\mathrm{CM}$, Abbott RD, Sharp DS, Yano LK, et al. Glucose intolerance and 23-year risk of coronary heart disease and total mortality. The Honolulu Heart Program. Diabetes Care 1999;22:1262-5.

10. The DECODE Study Group, on behalf of the European Diabetes Epidemiology Group. Glucose tolerance and mortality: Comparison of WHO and American Diabetes Association diagnostic criteria. Lancet 1999:354:617-21.

11. Malmberg K, for the DIGAMI Study Group. Prospective randomized study of intensive insulin treatment on longterm survival after acute myocardial infarction in patients with diabetes mellitus. BMJ 1997:314:1512-5.

12. Shichiri M, Kishikawa $H$, Ohkubo $Y$, Wake N. Long-term results of the Kumamoto Study on optimal diabetes control in type 2 diabetic patients. Diabetes Care 2000;23(suppl. 2):B21-9. 
13. Hanefeld $M$, Cagatay $M$, Petrowitsch T, Neuser D, Petzinna D, Rupp M. Acarbose reduces the risk for myocardial infarction in Type 2 diabetic patients: Meta-analysis of seven long-term studies. Eur Heart J 2004;25: 10-6.

14. Fontbonne $A$, Eschwège $E$, Cambien F, Richard J-L, Ducimetière $P$, Thibult $N$, et al. Hypertriglyceridemia as a risk factor of coronary heart disease mortality in subjects with impaired glucose tolerance or diabetes. Results from the 11-year follow-up of the Paris Prospective Study. Diabetologia 1989;32:300-4.

15. Beks PHJ, Mackaay AJC, De Vries H, De Neeling JND, Bouter LM, Heine RJ. Carotid artery stenosis is related to blood glucose level in an elderly Caucasian population: The Hoorn Study. Diabetologia 1997;40:290-8.

16. Del Prato S. In search of normoglycaemia in diabetes: Controlling postprandial glucose. Intern J Obes 2002;26(suppl. 1):S9-17.

17. Heine RJ, Balkau B, Ceriello A, Del Prato S, Horton ES, Taskinen M-R. What does postprandial hyperglycaemia mean? Diabetic Med 2004;21:208-13.

18. Davies MJ. Post-prandial hyperglycaemia and prevention of cardiovascular disease. Diabetic Med 2005;22(suppl. 1):6-9.

19. De Venciana M, Major CA, Morgan MA, Asrat T, Toohey JS, Lien JM, et al. Postprandial versus preprandial blood glucose monitoring in women with gestational diabetes mellitus requiring insulin therapy. N Engl J Med 1995;333:1237-41.

20. Avignon A, Radauceanu A, Monnier L. Nonfasting plasma glucose is a better marker of diabetic control than fasting plasma glucose in type 2 diabetes. Diabetes Care 1997;20:1822-6.

21. Soonthorpun S, Rattarasarn C, Leelawattana R, Setasuban W. Postprandial plasma glucose: A good index of glycemic control in type 2 diabetic patients having near-normal fasting glucose levels. Diabetes Res Clin Pract 1999:46:23-7.

22. Bonora $E$, Formentini $G$, Calcaterra $F$, Bonadonna RC, Lombardi $S$, Muggeo $M$, et al. Plasma glucose levels throughout the day and $\mathrm{HbA}_{1 \mathrm{C}}$ interrelationships in type 2 diabetes: Implications for treatment and monitoring of metabolic contol. Diabetes Care 2001;24:2023-9.

23. Monnier L, Lapinski $\mathrm{H}$, Colette $\mathrm{C}$. Contributions of fasting and postprandial plasma glucose increments to the overall diurnal hyperglycemia of type 2 diabetic patients: Variations with increasing levels of $\mathrm{HbA}_{1 \mathrm{c}}$. Diabetes Care 2003:26:881-5.

24. Norman GR, Streiner DL. Biostatistics: The bare essentials. St. Louis: Mosby-Year Book; 1994.

25. Monnier L, Colette C, Rabasa-Lhoret $R$, Lapinski $H$ Caubel C, Avignon A, et al. Morning hyperglycemic excursions: A constant failure in the metabolic control of non-insulin-using patients with type 2 diabetes. Diabetes Care 2002;25:737-41

26. Molnar GD, Taylor WF, Langworthy A. On measuring the adequacy of diabetes regulation: Comparison of continuously monitored blood glucose patterns with values at selected time points. Diabetologia 1974; 10:139-43.
27. Bolli GB. The dawn phenomenon: Its origin and contribution to early morning hyperglycemia in diabetes mellitus. Diabetes Metab (Paris) 1988; 14:675-86.

28. Lerman-Garber I, López-Ponce A, Flores RAM, Brito-Córdova GX, Velasco-Pérez ML, Villa AR, et al. Comparing easy and accessible parameters of glycemic control in type 2 diabetes. Rev Invest Clínica 2001;53:18-25.

29. Trovati M, Burzacca S, Mularoni E, Massucco P, Cavalot F, Mattiello L, et al. Occurrence of low blood glucose concentrations during the afternoon in Type 2 (noninsulin-dependent) diabetic patients on oral hypoglycaemic agents: Importance of blood glucose monitoring. Diabetologia 1991;34:662-7.

30. Bouma M, Kriegsman DM, Dekker JH, Kostense PJ, Sonnaville JJ, Heine RJ, et al. How valid is fasting plasma glucose as a parameter of glycemic control in non-insulinusing patients with type 2 diabetes? Diabets Care 1999;22:904-7.

31. American Diabetes Association. Postprandial blood glucose. Consensus Statement. Diabetes Care 2001;24:7758.

32. Schmitz $O$, Juhl $C B$, Lund $S$, Djurhuus $C B$, Nyholm B, Pørksen $\mathrm{N}$, et al. $\mathrm{HbA}_{1 \mathrm{C}}$ does not reflect prandial plasma glucose excursions in type 2 diabetes (letter). Diabetes Care 2000;23:1859-60.

33. Hillman N, Herranz L, Grande C, Villaroel A, Pallardo LF. Is $\mathrm{Hb} \mathrm{A}_{1 \mathrm{C}}$ influenced more strongly by preprandial or postprandial glycemia in type 1 diabetes (letter)? Diabetes Care 2002:25:1100-1.

34. Hillman N, Herranz L, Grande C, Vaquero PM, Pallardo LF. What is the relative contribution of blood glucose levels at different time points of the day to $\mathrm{HbA}_{1 \mathrm{c}}$ in Type 1 diabetes? Diabetic Med 2004:21:468-70.

35. Trovati M, Burzacca S, Mularoni E, Massucco P, Cavalot $\mathrm{F}$, Mattiello $\mathrm{L}$, et al. A comparison of the predictive power for overall blood glucose control of a "good" fasting level in Type diabetic patients on diet or with oral agents. Diabetic Med 1991;9:134-7.

36. Jeffcoate SL. Diabetes control and complications: The role of glycated haemoglobin, 25 years on. Diabetic Med 2004:21:657-65.

37. Faure E, Pallardo LF, Mesa J, Puig-Domingo M, GarcíaMayor R, Benito P, et al., for the MIDIA Study Group. $\mathrm{HbA}_{1 \mathrm{c}}$ and glycemic profile, basal- and post-treatment with miglitol, in an area with a Mediterranean diet (letter). Diabetes Care 2002;25: 1896-8.

\section{Endereço para correspondência:}

Walkyria de Paula Pimenta

Departamento de Clínica Médica

Faculdade de Medicina de Botucatu - UNESP

18618-970 Botucatu, SP

Fax: (14) 3882-2238

E-mail: wpimenta@fmb.unesp.br 